\title{
Interfacial mass transfer by controlled multilayer disassembly $\dagger$
}

Cite this: Chem. Commun., 2014 50, 8154

Received 20th January 2014, Accepted 19th May 2014

DOI: $10.1039 / \mathrm{c} 4 \mathrm{cc} 00495 \mathrm{~g}$

www.rsc.org/chemcomm

\author{
Michael Morozov, $\ddagger^{\mathrm{a}}$ Leila Motiei, $\ddagger^{\mathrm{a}}$ Joyanta Choudhury, $\S^{\mathrm{a}}$ Antonino Gulino, ${ }^{b}$ \\ Michal Lahav ${ }^{a}$ and Milko E. van der Boom*a
}

\begin{abstract}
We demonstrated the one-pot disassembly of self-propagating molecular assemblies (SPMAs) by ligand exchange and the subsequent covalent binding of the molecular components to other surfaces. These functionalized surfaces are suitable for regenerating the SPMAs.
\end{abstract}

Electron and mass-transfer phenomena are of great significance in biological processes. Whereas electron-transfer has been studied in detail in many artificial systems, ${ }^{1}$ mass-transfer between interfaces is relatively rare. ${ }^{2}$ This is somewhat surprising given the fact that mass-transfer between membranes is essential for life. Moreover, interfacial mass-transfer could lead to thin film self-replication on solid surfaces as demonstrated by Kiedrowski. ${ }^{3}$ A seminal example of mass-transfer between interfaces is Rebek's three-phase test, which is a polymer-based method to detect intermediates in various reactions. ${ }^{4}$ In this report, we demonstrate a specially designed system to transfer metal complexes between interfaces mediated by liquid media. The structural features and properties of a redox-active monolayer can be "copied" to another surface by the disassembly of a metal-organic network.

We have recently reported the formation of self-propagating molecular assemblies (SPMAs) on $\mathrm{SiO}_{x}$ and on Indium-Tin-Oxide (ITO)coated glass. $^{5-7}$ These assemblies are coordination-based networks of osmium $(1,2)$, ruthenium (3), or cobalt polypyridyl complexes crosslinked via a palladium or platinum salt (Scheme 1 and 2).

The formation of the SPMAs used in this study involves three steps: (a) hydrophilic substrate surfaces are functionalized with a benzyl-chloride terminated coupling layer (CL) ${ }^{8}$ using siloxane chemistry, (b) the CLs are reacted from solution at elevated temperatures with polypyridyl complex (1) affording template layers (TLs) consisting of covalently bound pyridinium salts of complex 1 that can bind metal salts, and (c) the iterative exposure

\footnotetext{
${ }^{a}$ Department of Organic Chemistry, The Weizmann Institute of Science, Rehovot 7610001, Israel. E-mail: milko.vanderboom@weizmann.ac.il

${ }^{b}$ Dipartimento di Scienze Chimiche, Università di Catania, Catania, Italy

$\dagger$ Electronic supplementary information (ESI) available: Experimental procedures and characterization data. See DOI: 10.1039/c4cc00495g

\$ These authors contributed equally.

$\S$ Current address: Department of Chemistry, IISER Bhopal, India.
}

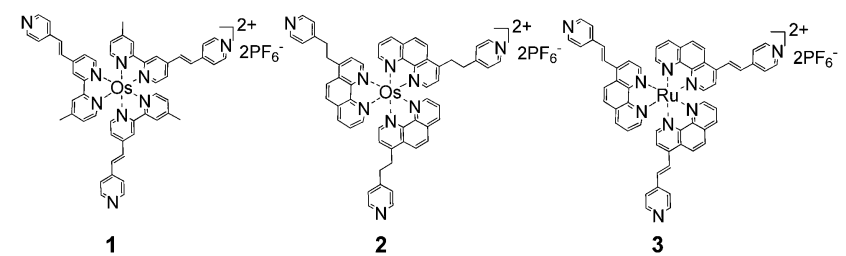

Scheme 1 Chemical structures of the polypyridyl complexes (1-3). ${ }^{5,7}$

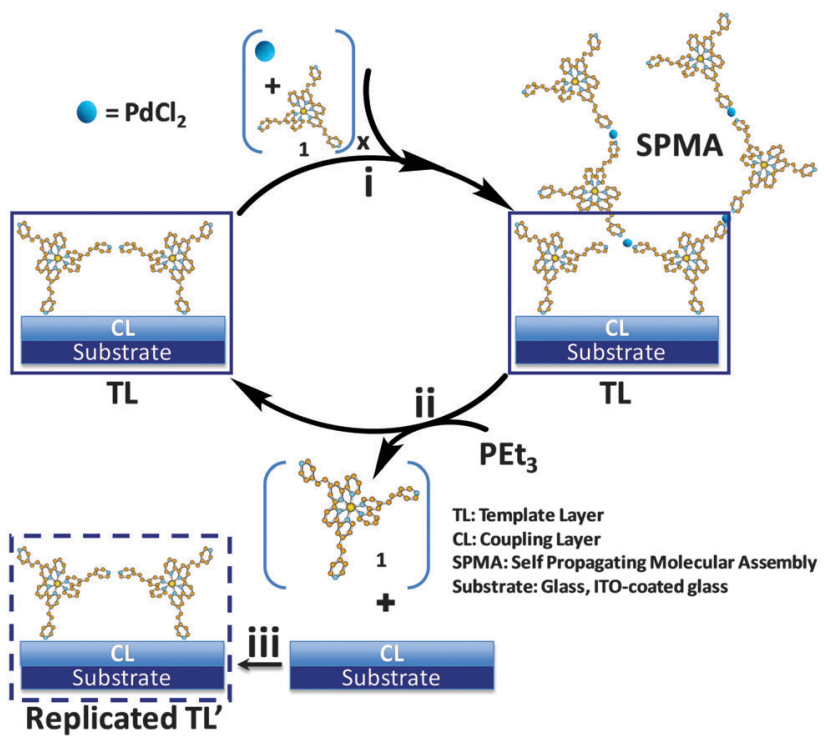

Scheme 2 Schematic presentation of the mass-transfer process between molecular interfaces. (i) SPMA formation by alternating depositions of a Pd salt and a polypyridyl complex (1) on a template layer (TL); $x=$ number of deposition steps. ${ }^{5,7}$ (ii) Disassembly of the SPMA with $\mathrm{PEt}_{3}$ (triethylphosphine) to regenerate the $T L$ with subsequent release of the complexes in solution. (iii) Reaction of the complexes (1) released to the solution with covalently bound chlorobenzyl-terminated coupling layers (CLs) to afford replicated TL's, which can be used to generate SPMAs.

of these TLs to solutions of a polypyridyl complex (1-3) with multiple metal-binding sites and $\mathrm{PdCl}_{2}(\mathrm{PhCN})_{2}$ (Scheme S1, ESI $\dagger$ and Scheme 2, step i). 
The physicochemical properties of the SPMAs increase exponentially with the number of deposition steps. ${ }^{5,7}$ The exponential growth results from the storage of an excess of palladium salt inside these films. During immersion of the SPMAs in a solution of a polypyridyl complex, the excess of palladium aids the coordination of polypyridyl complexes beyond a single molecular layer. Consequently, more palladium can be stored upon increasing the thickness of the SPMAs, which again leads to a larger increase in the thickness in the next deposition step. The electron density and roughness remains nearly constant upon film growth. Linear growth can be achieved by reducing the amount of palladium.

In a typical disassembly and mass transfer experiment, presented in Scheme 2, several ITO $(0.7 \mathrm{~cm} \times 5 \mathrm{~cm} \times 0.7 \mathrm{~mm})$ and glass substrates $(1 \mathrm{~cm} \times 2 \mathrm{~cm} \times 0.1 \mathrm{~cm})$ functionalized with a SPMA, consisting of 11 depositions of $\mathrm{PdCl}_{2}\left(\mathrm{PhCN}_{2}\right.$ and complex 1, and ITO substrates functionalized with a CL were placed in a Teflon sample holder. The distance between the surface planes was fixed at $0.25 \mathrm{~cm}$ to $2 \mathrm{~cm}$, which is several orders of magnitude larger than the thickness of the molecular interfaces used (SPMA: $16 \mathrm{~nm}$; CL: $0.8 \mathrm{~nm}$ ). Then, the set-up was transferred into a glass pressure vessel and immersed in a dry acetonitrile/toluene $(1: 1, \mathrm{v} / \mathrm{v})$ solution containing an excess of triethylphosphine $\left(\mathrm{PEt}_{3} ; 0.1 \mathrm{M}\right)$. Then the vessel was sealed and heated for $72 \mathrm{~h}$ at $90{ }^{\circ} \mathrm{C}$ under an inert atmosphere. Subsequently, the vessel was allowed to attain room temperature and the substrates were thoroughly washed with acetonitrile, acetone, and ethanol. UV/vis spectroscopy of the light brown solution indicated the presence of complex 1 (Fig. S1, ESI $\dagger$ ).

The thin films (TLs and SPMAs) were analyzed by optical (UV/vis) spectroscopy (Fig. 1A and B), cyclic voltammetry (CV; Fig. 1C and D), and X-ray photoelectron spectroscopy (XPS). These measurements indicate the complete disassembly of the 1-based SPMA and the formation of new TLs by interfacial mass-transfer of complex 1 and the subsequent reaction of $\mathbf{1}$ with the CL-functionalized substrates (Scheme 2, steps ii and iii). The UV/vis spectra of the TLs are similar and exhibit the characteristic singlet and triplet metal-toligand charge-transfer (MLCT) bands at $\lambda=500 \mathrm{~nm}$ and $\lambda=$ $700 \mathrm{~nm}$ of complex 1 . The absorption intensities are similar to TLs obtained independently by the reaction of complex 1 with a CL (Fig. 1A). Cyclic voltammograms of these 1-based TLs on ITO substrates showed a reversible redox process, characteristic of an $\mathrm{Os}^{2+/ 3+}$ couple (Fig. 1C). Whereas there is some variation in the wave shapes, the half-wave potential values are in the expected range of $0.93 \pm 0.1 \mathrm{~V}$ (Table S1, ESI + ). The peak current is directly proportional to the scan rate within the range of 0.1 and $1 \mathrm{~V} \mathrm{~s}^{-1}$, indicating a surface-confined process that is not limited by diffusion (Fig. 1D). The estimated surface coverage, $\Gamma$, ranges from $1.2 \times 10^{-10}$ to $1.4 \times$ $10^{-10} \mathrm{~mol} \mathrm{~cm}^{-2}$ (Table S1, ESI $\dagger$ ), indicating the presence of a dense structure. ${ }^{7}$ XPS analysis revealed the presence of a pyridinium salt by quaternization of one pyridyl moiety of complex $1 ., 7,9$ The pyridinium and pyridyl $\mathrm{N}$ 1s signals were observed in a $1: 8$ ratio at 400 and $401.5 \mathrm{eV}$, respectively. No palladium was observed. The good agreement among the UV/vis, electrochemical, and XPS data for all TLS confirms that it remains bound to the substrate surface after the disassembly of the SPMA and that the newly generated TLs have essentially the same structure as the TL used to build-up the SPMA. To confirm the structural similarity and to evaluate the function of
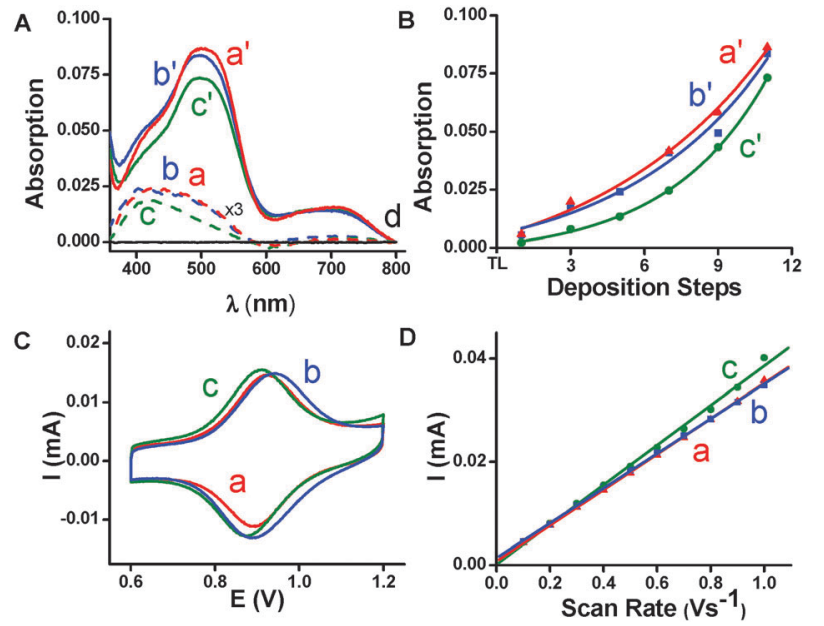

Fig. 1 Optical (UV/vis) spectroscopy (A, B) and cyclic voltammetry (CV) (C, D) data. (A) Absorption spectra of (a) TL used for the built-up of a 1-based SPMA, (b) TL generated after disassembly of the 1-based SPMA with PEt 3 , (c) replicated TL'. (a-c; enlarged $3 \times$ for clarity), and $\left(a^{\prime}-c^{\prime}\right)$ are the 1 -based SPMAs on ITO-coated glass substrates functionalized with ( $\left.a^{\prime}\right)$ TL formed by reacting a $C L$ with complex $1,\left(b^{\prime}\right) T L$ after disassembly of the 1-based SPMA with $\mathrm{PEt}_{3},\left(\mathrm{C}^{\prime}\right)$ replicated $\mathrm{TL}^{\prime}$, (d) baseline. (B) Exponential correlation between the intensities of the MLCT bands $(\lambda \approx 500 \mathrm{~nm})$ and the number of complex 1 deposition steps for the SPMAs $\left(a^{\prime}-c^{\prime}\right)$ built on the three TLs (a-c). (C) Cyclic voltammograms of TLs $(a-c)$. The scan rate is $400 \mathrm{mV} \mathrm{s}^{-1}$. (D) Plot of peak currents as a function of the scan rate of the TLs $(a-c)$, with $R^{2}>0.98$ for the linear fits (see ESI $\dagger$ for Experimental details).

the TLs, we regenerated the 1-based SPMA on the TLs that were obtained after the disassembly process. In parallel, we also built this SPMA on the replicated TL's. The nearly identical UV/vis spectra (Fig. 1A spectra $\mathrm{a}^{\prime}-\mathrm{c}^{\prime}$ ) and the similar SPMA growth behavior obtained after the deposition of 11 depositions of $\mathrm{PdCl}_{2}\left(\mathrm{PhCN}_{2}\right.$ and complex 1 confirm the efficient interfacial transfer of complex 1 (Fig. 1B), and the structural and functional similarities of the original TLs and replicated TL's. These results are not obvious because the overall process also involves the release of the palladium salt and a reactive phosphine that also might have reacted with the CLs. ${ }^{10}$ Reaction of $\mathrm{PEt}_{3}$ with the CLs under the same conditions as the mass-transfer experiments resulted in the formation of some surface-bound phosphonium salt ( $10 \%$ by XPS). Apparently, sufficient benzyl chloride moieties remain for complexes 1-3 to bind to the CLs.

We further demonstrated selective transfer of one type of pyridyl complex to ITO substrates functionalized with CLs by the onepot disassembly of 2- and 3-based SPMAs on glass substrates with $\mathrm{PEt}_{3}$ (Scheme 1; Fig. S2, ESI $†$ ). Different metals (2: osmium, and 3: ruthenium) were used to accurately determine the composition of the replicated TL's by electrochemistry and XPS. Although the structures of these complexes are similar, we tuned the reactivity of the terminal pyridine groups by using $\mathrm{C}-\mathrm{C}(2) v s . \mathrm{C}=\mathrm{C}(3)$ moieties. T These experiments were carried out under the same conditions used for the disassembly of the 1-based SPMA and the formation of the replicated 1-based TL' (Scheme 2).

Interestingly, the formed TLs consist of osmium complex 2, although our data indicate that both 2- and 3-based SPMAs undergo disassembly upon reaction with $\mathrm{PEt}_{3}$. The $\mathrm{CV}$ of the replicated TL' shows clearly one reversible redox process (Fig. 2A). 

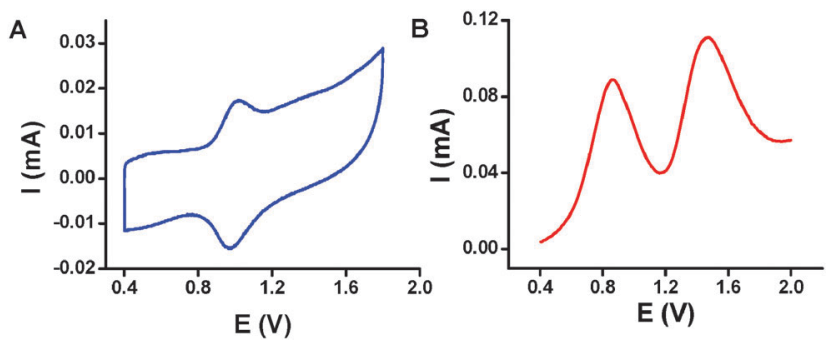

Fig. 2 (A) Representative cyclic voltammogram (CV) of the replicated 2-based TL' formed by disassembly of 2- and 3-based SPMAs with PEt ${ }_{3}$ and selective transfer of osmium complex $\mathbf{2}$ to ITO substrates functionalized with CLs. The scan rate is $400 \mathrm{mV} \mathrm{s}^{-1}$. (B) Differential pulse voltammogram (DPV) of the reaction solution after the disassembly of $\mathbf{2}$ - and 3 -based SPMAs with PEt 3 . The following conditions were applied: scan rate: $20 \mathrm{mV} \mathrm{s}^{-1}$, pulse width: $16 \mathrm{~ms}$, pulse amplitude: $50 \mathrm{mV}$, and pulse period, $200 \mathrm{~ms}$. For both (A) and (B) electrolyte: $0.1 \mathrm{M} \mathrm{TBABF} / \mathrm{CH}_{3} \mathrm{CN}$ was used at room temperature. Pt wire and $\mathrm{Ag} / \mathrm{AgCl}$ were used as counter and reference electrodes, respectively. The functionalized ITO-coated glass substrates were used as working electrodes.

The redox couple at $E_{1 / 2}=0.85 \mathrm{~V} v s . \mathrm{Ag} / \mathrm{AgCl}$ is characteristic for the presence of an interface consisting of osmium complexes (2) $\left(\Gamma \approx 2.1 \times 10^{-11} \mathrm{~mol} \mathrm{~cm}{ }^{-2}\right)$. No signal was apparent indicating the presence of ruthenium complex (3). Differential pulse voltammetry (DPV) of the reaction solution shows that both complexes are stable under the applied reaction conditions and are available for reacting with the CLs (Fig. 2B). The two potential peaks at $0.85 \mathrm{~V}$ and $1.46 \mathrm{~V}$ vs. $\mathrm{Ag} / \mathrm{AgCl}$ are characteristic of complexes 2 and 3, respectively.

Reacting only complex 2 or $3(0.2 \mathrm{mM})$ for $72 \mathrm{~h}$ at $90{ }^{\circ} \mathrm{C}$ with ITO substrates functionalized with CLs immersed in dry toluene/ acetonitrile $(1: 1, \mathrm{v} / \mathrm{v})$ resulted in the formation of the corresponding TLs, as judged by electrochemical analyses (Fig. S3, ESI $\dagger$ ). Apparently, the reactivity of complex 3 is sufficiently high to form a TL (in the absence of complex 2). The CV of the 2-based TL is nearly identical to the replicated TL' (Fig. S3A, ESI $\dagger$ ).

The selective generation of the 2-based TL was also indicated by XPS measurements. An interface of $1.5 \mathrm{~nm}$ was observed on the ITO substrate having an elemental ratio of $\mathrm{N}: \mathrm{Os}=10: 1$, which is in good agreement with the expected ratio $(9: 1)$. In addition, no signal indicative of ruthenium complex (3) was observed. The pyridinium and pyridyl $\mathrm{N}$ 1s signals for the 2-based TL were observed at 401.9 and $400.2 \mathrm{eV},{ }^{5,7,9}$ respectively, with an elemental ratio $\mathrm{N}^{+}: \mathrm{N}=1: 9$ (expected $1: 8$ ). Apparently, the complexes are covalently bound to the CL with one of the pyridine groups.

In summary, we showed that interfacial transfer of metal complexes can be achieved and triggered using a metal exchange process that occurs between different ligands (i.e., $\mathrm{PEt}_{3}$ and substituted pyridines) at the boundaries of a solution-solid state interface. This metal-ligand process is likely to be highly complex and involves reversible steps. The transfer and subsequent covalent binding of the polypyridyl complexes takes place from a medium that contains an excess of other reactive species including $\mathrm{PEt}_{3}$ and metal salts thereof. Nevertheless, this process is highly selective and can even proceed in the presence of an equimolar amount of another polypyridyl complex. The overall structure and functionality of the replicated templates is identical to the templates used for their formation. To this end, the coupled disassembly-assembly processes ${ }^{11}$ of metal-organic networks presented here are examples of System Chemistry involving solid interfaces. Such processes might provide fundamental insight into signaling, communication and molecular replication. ${ }^{12}$

This research was supported by the Helen and Martin Kimmel Center for Molecular Design, the Jacob and Charlotte Lehrman Foundation, and Luis Stuhlberger, Brazil. MvdB is the incumbent of the Bruce A. Pearlman Professorial Chair in Synthetic Organic Chemistry. We thank Dr Petr Milko (WIS) for performing the DFT calculations.

\section{Notes and references}

I DFT calculations indicate that the electron density of the pyridine moieties of complexes $\mathbf{1}$ and $\mathbf{3}$ is nearly identical ( $Q$ (pyr) is 0.00 and 0.01 , respectively), whereas these moieties exhibit a higher electron density for complex 2 ( $Q(\mathrm{pyr})$ is -0.05$)$.

1 Electron Transfer in Chemistry, ed. V. Balzani, Wiley-VCH Verlag $\mathrm{GmbH}, 2008$.

2 R. A. Bissell, E. Cordova, A. E. Kaifer and J. F. Stoddart, Nature, 1994, $369,133$.

3 A. Luther, R. Brandsch and G. von Kiedrowski, Nature, 1998, 396, 245.

4 J. Rebek and F. Gavina, J. Am. Chem. Soc., 1974, 96, 7112.

5 G. de Ruiter, M. Lahav, G. Evmenenko, P. Dutta, D. A. Cristaldi, A. Gulino and M. E. van der Boom, J. Am. Chem. Soc., 2013, 135, 16533; L. Motiei, M. Feller, G. Evmenenko, P. Dutta and M. E. van der Boom, Chem. Sci., 2012, 3, 66; J. Choudhury, R. Kaminker, L. Motiei, G. de Ruiter, M. Morozov, F. Lupo, A. Gulino and M. E. van der Boom, J. Am. Chem. Soc., 2010, 132, 9295; L. Motiei, M. Altman, T. Gupta, F. Lupo, A. Gulino, G. Evmenenko, P. Dutta and M. E. van der Boom, J. Am. Chem. Soc., 2008, 130, 8913.

6 For examples of surface-bound metal-organic assemblies see: J. Poppenberg, S. Richter, C. H. H. Traulsen, E. Darlatt, B. Baytekin, T. Heinrich, P. M. Deutinger, K. Huth, W. E. S. Unger and C. A. Schalley, Chem. Sci., 2013, 4, 3131; D. Zacher, R. Schmid, C. Wöll and R. A. Fischer, Angew. Chem., Int. Ed., 2011, 50, 176; K. Kanaizuka, R. Haruki, O. Sakata, M. Yoshimoto, Y. Akita and H. Kitagawa, J. Am. Chem. Soc., 2008, 130, 15778.

7 L. Motiei, M. Lahav, A. Gulino, M. A. Iron and M. E. van der Boom, J. Phys. Chem. B, 2010, 114, 14283.

8 D. Li, M. A. Ratner, T. J. Marks, C. Zhang, J. Yang and G. K. Wong, J. Am. Chem. Soc., 1990, 112, 7389.

9 A. Gulino, Anal. Bioanal. Chem., 2013, 405, 1479.

10 J. S. Mugridge, G. Szigethy, R. G. Bergman and K. N. Raymond, J. Am. Chem. Soc., 2010, 132, 16256; M. Jung, Y. Suzaki, T. Saito, K. Shimada and K. Osakada, Polyhedron, 2012, 40, 168.

11 X. Li, X. Zhao, J. Zhang and Y. Zhao, Chem. Commun., 2013, 49, 10004; J. M. A. Carnall, C. A. Waudby, A. M. Belenguer, M. C. A. Stuart, J. J.-P. Peyralans and S. Otto, Science, 2010, 327, 1502; J. Baram, E. Shirman, N. Ben-Shitrit, A. Ustinov, H. Weissman, I. Pinkas, S. G. Wolf and B. Rybtchinski, J. Am. Chem. Soc., 2008, 130, 14966; E. Menozzi, R. Pinalli, E. A. Speets, B. J. Ravoo, E. Dalcanale and D. N. Reinhoudt, Chem. - Eur. J., 2004, 10, 2199; G. Ashkenasy, A. Ivanisevic, R. Cohen, C. E. Felder, D. Cahen, A. B. Ellis and A. Shanzer, J. Am. Chem. Soc., 2000, 122, 1116; I. Doron-Mor, A. Hatzor, A. Vaskevich, T. van der Boom-Moav, A. Shanzer, I. Rubinstein and H. Cohen, Nature, 2000, 406, 382; A. Hatzor, T. van der Boom-Moav, S. Yochelis, A. Vaskevich, A. Shanzer and I. Rubinstein, Langmuir, 2000, 16, 4420.

12 L. E. Orgel, Nature, 1992, 358, 203. 